\title{
Resonance-enhanced below-threshold harmonic generation of He atoms in few-cycle laser fields
}

\section{Peng-Cheng Li**广}

Center for Quantum Science and Engineering, and Center for Advanced Study in Theoretical Sciences, Department of Physics, National Taiwan University, Taipei 10617, Taiwan

College of Physics and Electronic Engineering, Northwest Normal University, Lanzhou 730070, China

E-mail: lipc@nwnu.edu.cn

\section{Shih-I Chu}

Center for Quantum Science and Engineering, and Center for Advanced Study in Theoretical Sciences, Department of Physics, National Taiwan University, Taipei 10617, Taiwan

E-mail: sichu@ku.edu

\begin{abstract}
We study resonance-enhanced harmonic generation of He atoms below the ionization threshold in few-cycle infrared laser fields. An accurate angular-momentum-dependent model potential is constructed for the description of the He atoms low-lying and Rydberg states. High-order harmonic generation (HHG) is obtained by solving the time-dependent Schrödinger equation, and effects of phase matching are considered by solving the Maxwell wave equation. We find that the yield of the harmonic 13 on resonant is largely increased.
\end{abstract}

2013 International Workshop on Computational Science and Engineering,

*Speaker.

$\dagger$ This work was partially supported by National Science Council and National Taiwan University (Grant No. 102R8700-2 and 102R104021). PCL is partially supported by National Natural Science Foundation of China (Grant No. 11047016, and 11264036, and 11364039) and Natural Science Foundation of Gansu Province of China (Grant No. 1308RJZA195). 
High-order harmonic generation (HHG) by the interaction of an intense laser field with an atomic or molecular target, leading to the production of the coherent extreme-ultraviolet (XUV) and attosecond pulses, has attracted much interest in the subject of ultrafast science and technology in the last decade $[1,2,3,4,5]$. The essential features in HHG, such as the plateau where all the harmonics have the similar amplitude and cutoffs have been well understood by the semiclassical three-step model [6]. It is found that the cutoff occurs approximately at the energy $I_{p}+3.17 U_{p}$, where $I_{p}$ is the atomic ionization potential, $U_{p}$ is the ponderomotive potential. Furthermore, the HHG above the ionization threshold has been widely investigated by means of the strong field approximation (SFA) [7]. However, in SFA theory, the intermediate bound states and the Coulomb interaction in the final state are completely neglected, so it is not accurate as a method to describe the below-threshold HHG. In this paper, we present an ab initio study of below-threshold harmonic generation by solving the time-dependent Schrödinger equation (TDSE) in space and time by means of the time-dependent generalized pseudospectral method (TDGPS) [8], and propagation effects are considered by solving the three dimensional (3D) Maxwell wave equation [9, 10], which allow us to obtain the phase-matched below-threshold HHG. In particular, we have chosen $\mathrm{He}$ atoms as atomic medium for below-threshold harmonic generation due to its single-electron transition energies for $1 s^{2}-1 s 2 p^{1} P(21.22 \mathrm{eV})$ and $1 s^{2}-1 s 3 p{ }^{1} P(23.09 \mathrm{eV})$ coincide with the harmonic 13 (H13) and 15 (H15) photon energies of the Ti: Sapphire laser. Furthermore, the He atom shows the absence of inner-shell absorption, and allows us to well understand the dynamics in resonance-enhanced harmonic generation.

HHG is produced by the interaction of an intense laser field with a single atom can be calculated by solving the following TDSE (in atomic units),

$$
i \frac{\partial \psi(\mathbf{r}, t)}{\partial t}=\hat{H} \psi(\mathbf{r}, t)=\left[\hat{H}_{0}+\hat{V}(\mathbf{r}, t)\right] \psi(\mathbf{r}, t) .
$$

Here, $\hat{V}(\mathbf{r}, t)$ is the time-dependent atom-field interaction, and $\hat{H}_{0}$ represents unperturbed atom Hamiltonian, it can be written as

$$
\hat{H}_{0}=-\frac{1}{2} \nabla^{2}+\sum_{l}\left|Y_{l}^{0}>V_{l}<Y_{l}^{0}\right|,
$$

where $V_{l}$ is the model potential for $\mathrm{He}$ and $Y_{l}^{0}$ is a spherical harmonic. In order to obtain the accurate calculation of the harmonic spectra of $\mathrm{He}$, an angular-momentum-dependent model potential is constructed as the following form:

$$
V_{l}=-\frac{1}{r}-\frac{\alpha}{2 r^{4}} W_{6}\left(\frac{r}{r_{c}}\right)-\left(\frac{N-S}{r}+A_{1}\right) e^{-B_{1} r}-\left(\frac{S}{r}+A_{2}\right) e^{-B_{2} r},
$$

where $\alpha$ is the $\mathrm{He}^{+}$core dipole polarizability $[11,12], W_{6}$ is a core cutoff function given by

$$
W_{n}(x)=1-\left[1+n x+\frac{(n x)^{2}}{2 !}+\ldots+\frac{(n x)^{2}}{n !}\right] e^{-n x},
$$

and $r_{c}$ is an effective $\mathrm{He}^{+}$core radius. In the present work we find it is sufficient to use five different angular-momentum-dependent model potential. Namely, all for states with $l=0,1,2,3$ and $l \geq 3$. The HHG spectrum in the single-atom level by the Fourier transformation of time-dependent 


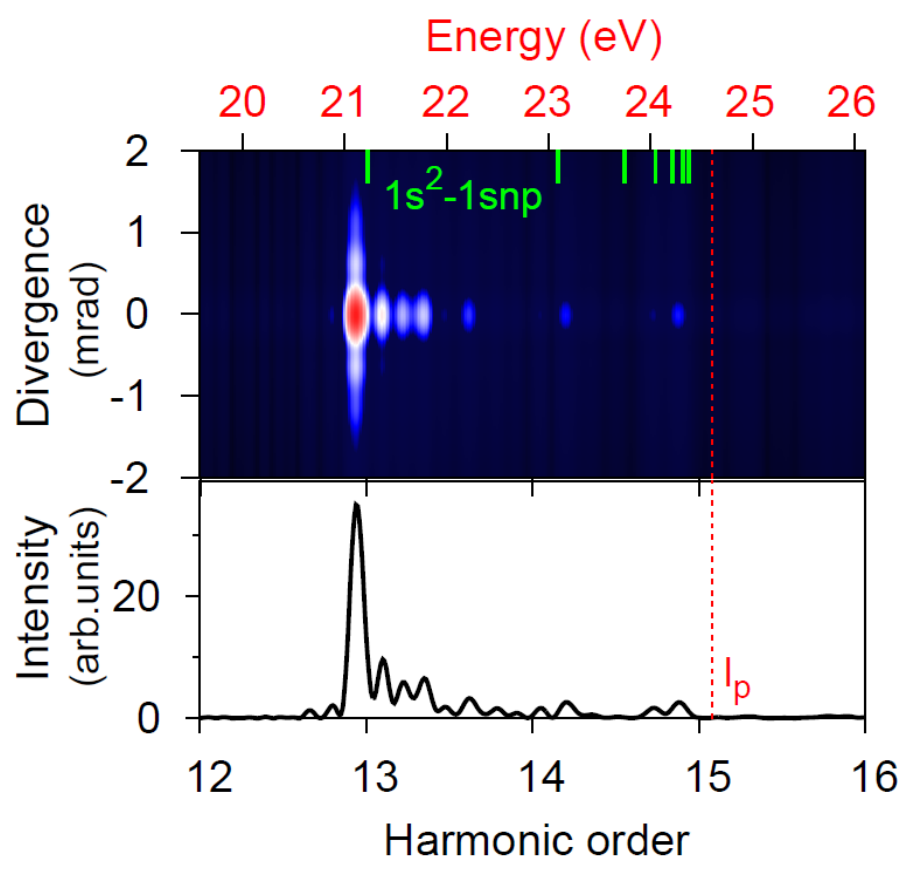

Figure 1: Below-threshold harmonic spectra for harmonic 13-15 in He by a few-cycle 760-nm laser pulse. The laser peak intensity in the center of gas jet is $I=9.0 \times 10^{13} \mathrm{~W} / \mathrm{cm}^{2}$. The red dashed line indicate the ionization potential $I_{p}$.

dipole moment, and the propagation of the laser field and harmonic field in a macroscopic medium is described by the Maxwell wave equation [13, 14].

Figure 1 shows below-threshold harmonic spectra for harmonic 13-15 in He by a fewcycle 760-nm laser pulse. In calculation, the laser peak intensity in the center of gas jet is $I=$ $9.0 \times 10^{13} \mathrm{~W} / \mathrm{cm}^{2}$ (Multi-photon ionization regime, and Keldysh parameter $\gamma=\sqrt{I_{p} /\left(2 U_{p}\right)} \gg 1$ ). We found that the yield of the near H13 is enhanced significantly because it is just on resonance. Namely, the 13-photons absorption energy of the $760 \mathrm{~nm}$ just equal to the transition energy of the $1 s^{2}-1 s 2 p{ }^{1} P(21.22 \mathrm{eV})$, and its contributions is dominant. But the contribution from singleelectron transition for $1 s^{2}-1 s 3 p{ }^{1} P(23.09 \mathrm{eV}), 1 s^{2}-1 s 4 p{ }^{1} P(23.74 \mathrm{eV})$, and $1 s^{2}-1 s 5 p{ }^{1} P(24.05$ $\mathrm{eV}$ ) is small (The green solid line indicates the spectra lines). In addition, the below-threshold harmonic spectra for harmonic 13-15 as a function of the laser intensity and the photon energy in the single atom response have been shown in Fig.2. This result is obtained from a numerical solution of the time-dependent TDSE in space and time by means of the time-dependent generalized pseudospectral method (TDGPS) [8]. The result shows the yield of below-threshold harmonic spectra have strongly depend on the laser peak intensity, and the yield of the H13 which just on resonant from the transition of the $1 s^{2}-1 s 2 p^{1} P$ is always enhanced largely, and the yields of the near harmonic $\mathrm{H} 15$ only have a slight enhancement with the laser intensity.

In conclusion, we have investigated the resonance-enhanced below-threshold harmonic generation of He atoms in few-cycle laser fields. An accurate angular-momentum-dependent model potential is constructed for the description of the He atomic fine structure. We obtain accurate macroscopic below-threshold harmonic spectra by means of solving accurately the TDSE and Maxwell 


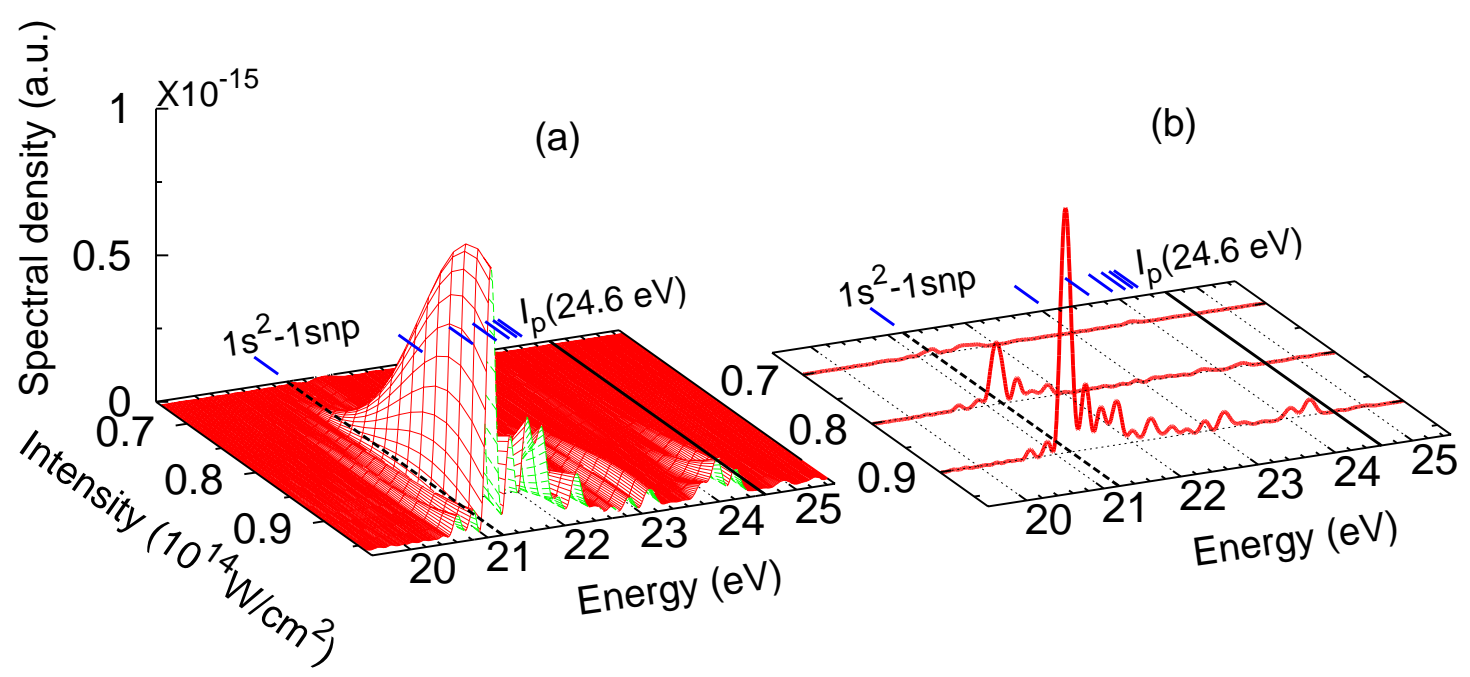

Figure 2: (a) Below-threshold harmonic spectra for harmonic 13-15 as a function of the laser intensity and the photon energy in the single atom response. (b) Same as (a) but for $I=7.0 \times 10^{13} \mathrm{~W} / \mathrm{cm}^{2}, I=$ $8.0 \times 10^{13} \mathrm{~W} / \mathrm{cm}^{2}$, and $I=9.0 \times 10^{13} \mathrm{~W} / \mathrm{cm}^{2}$, respectively. The black dashed lines in (a) and (b) indicate the transition energy of the $1 s^{2}-1 s 2 p{ }^{1} P(21.22 \mathrm{eV})$, and the black solid line indicates ionization potential $I_{p}$.

wave equation. It is shown that the yield of the $\mathrm{H} 13$ on resonant is largely increased, which is produced from the transition of the $1 s^{2}-1 s 2 p^{1} P(21.22 \mathrm{eV})$, and its contributions is dominant.

\section{References}

[1] E. Goulielmakis et al., Science 320, 1614 (2008).

[2] G. Sansone et al., Science 314, 443 (2006).

[3] J. Itatani et al., Nature 432, 867 (2004).

[4] R. Kienberger et al., Science 297, 1144 (2002).

[5] M. Drescher et al., Nature 419, 803 (2002).

[6] P. B. Corkum, Phys. Rev. Lett. 73, 1995 (1993).

[7] M. Lewenstein, P. Salières, and A. L'Huillier, Phys. Rev. A 52, 4747 (1995).

[8] X. M. Tong and S. I. Chu, Chem. Phys. 217, 119 (1997).

[9] M. B. Gaarde, J. L. Tate, and K. J. Schafer, J. Phys. B 41, 132001 (2008).

[10] V. Tosa, H. T. Kim, I. J. Kim, and C. H. Nam, Phys. Rev. A 71, 063807 (2005).

[11] X. Chu, S. I. Chu, and C. Laughlin, Phys. Rev. A 64, 013406 (2001).

[12] W.R. Johnson, D. Kolb, and K-N. Huang, At. Data Nucl. Data Tables 28, 333 (1983).

[13] C. Jin, A. T Le, C. A. Trallero-Herrero, and C. D. Lin, Phys. Rev. A 84, 043411 (2011).

[14] P. C. Li and S. I. Chu, Phys. Rev. A 86, 013411 (2012). 